\title{
Push-Pull Fatigue of the [110] and [410] Single Crystals of Iron
}

\author{
By Shozo Ikeda*
}

\begin{abstract}
Single crystals of iron with the [110] and [410] axes were fatigued in push-pull at constant total strain amplitudes in the range between 0.1 and $2 \%$. When the strain amplitude was smaller than $0.5 \%$, the cyclic hardening of the [410] crystal was similar to that of the [110] crystal. Clusters of edge dislocation dipoles and loops were developed parallel to the $\{112\}$ slip plane, which suggests the activation of single slip locally. When the strain amplitude is larger than $0.5 \%$, the cyclic hardening of the [410] crystal was much larger than that of the [110] crystal. Cell walls lie along symmetry planes with respect to the slip planes, which suggests multiple slip. Striation patterns on the fracture surfaces appeared parallel to the intersection between operative $\{112\}$ slip planes in both crystals. When a crack was forced to propagate to perpendicular to the slip vectors, in the [110] direction on the (110) plane, it propagated in the $\langle 332\rangle$ directions with a deviation of 40 degrees from the [110] direction
\end{abstract}

(Received October 19, 1978)

\section{Introduction}

Many studies have been made on the fatigue mechanism of single crystals of $f c c$ metals ${ }^{(1) \sim(9)}$. In the case of $b c c$ metals, however, such studies have been relatively few $^{(10) \sim(18)}$, especially those concerned with the effect of crystal orientations on the process to failure in pushpull fatigue ${ }^{(14)(15)(18)}$. In the present investigation, single crystals of iron oriented for multiple slip deformation are fatigued in push-pull, and cyclic hardening, shape changes, dislocation distributions, and fracture surfaces were observed. The results were analyzed in the light of a combination of operative slip systems in crystals under cyclic deformation.

\section{Experimental Procedure}

Test specimens $(2 \times 5 \times 24 \mathrm{~mm})$ oriented for two fold slip and four fold slip deformation were cut from a sheet of single crystals of iron. The axes of the prepared specimens deviated 4 and 13 degrees from the expected [110] and [100] direction respectively. These specimens will be denoted by [110] and [410] in this paper. The chemical composition in weight percent of the as-grown crystal is as follows:

\begin{tabular}{cccccccccc}
\hline $\mathrm{Si}$ & $\mathrm{Mn}$ & $\mathrm{P}$ & $\mathrm{S}$ & $\mathrm{Ni}$ & $\mathrm{Co}$ & $\mathrm{Cu}$ & $\mathrm{Al}$ & $\mathrm{O}$ & $\mathrm{C}$ \\
0.001 & 0.002 & 0.001 & 0.002 & 0.005 & 0.010 & 0.001 & 0.001 & 0.002 & 0.002 \\
\hline
\end{tabular}

After a mechanical polishing with emery paper, the surface of the gage section of $6 \mathrm{~mm}$ long was removed chemically by $50 \mu \mathrm{m}$ in a solution of fluoric acid $(5 \mathrm{~m} \ell)$ and hydroperoxide $(95 \mathrm{~m} \ell)$. Figure 1 shows the grips used in the present test. One of the grips slides along a guide with a frictional force less than $4 \mathrm{~N}$ (Newton) when no specimen was set. A closed loop electro-servo-hydraulic testing machine of $14 \mathrm{kN}$ capacity was used under grip

* National Research Institute for Metals, 2-3-12 Nakameguro Meguro-ku, Tokyo 153, Japan. distance control. The grip distance was measured by a differential transformer Shinko Electric DS-36. The total strain described in this paper means the relative displacement of the grips divided by the gage length. The plastic strain was calculated from the total strain minus the apparent elastic constant times the stress. $75 \mathrm{GN} / \mathrm{m}^{2}$ was used as the representative apparent elastic constant which was measured on the basis of the relation between the load against displacement using a specimen made by havily colled rolled iron; the flow stress of this specimen is much higher than the peak stress

1979 Vol. 20 


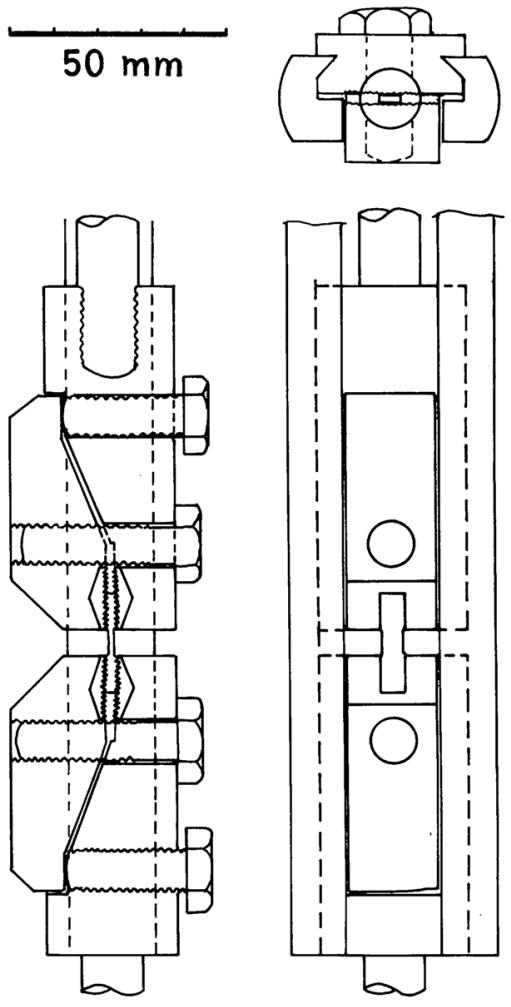

Fig. 1 Grips for tension-compression tests.

in the present test condition. All fatigue tests were carried out in air atmosphere at room temperature. Dislocation distributions were observed with a $500 \mathrm{kV}$ electron microscope. Fracture surfaces were observed with a scanning electron microscope.

\section{Experimental Results}

\section{Cyclic hardening}

The variation in peak stress with the number of cycles for the [110] and [410] crystals fatigued at various plastic strain amplitudes with a frequency of $0.5 \mathrm{~Hz}$ are shown in Fig. 2. The saturation stress of the [410] crystal was appreciably higher than that of the [110] crystal when the plastic strain amplitude was larger than $0.5 \%$. The saturation stress of both crystals deformed at a smaller strain amplitude of less than $0.5 \%$ were nearly the same. The cyclic softening was seen in the specimens cycled under a smaller strain amplitude of less

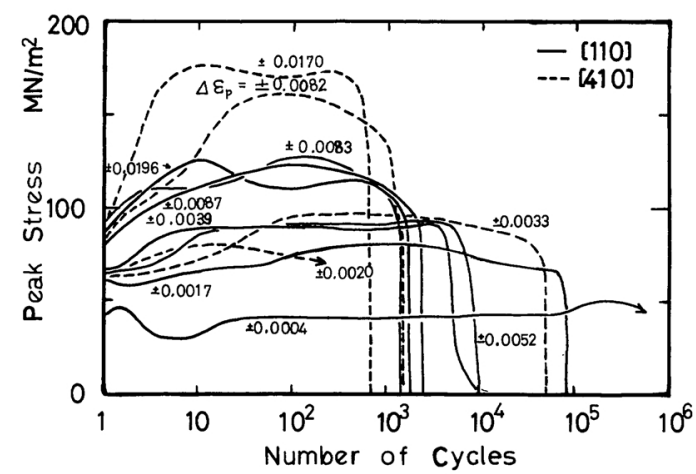

Fig. 2 The variation in peak stress with the number of cycles for the [110] and [410] crystals under various strain amplitudes and $0.5 \mathrm{~Hz}$.

than $0.2 \%$ in the initial stage. This behavior was caused by a successive increase in mobile dislocation density during cyclic deformation as pointed out by Yoshikawa and Okamoto ${ }^{(10)}$.

The saturation stress defined as the muximum peak stress of the each curve in Fig. 2 are plotted against the total strain amplitude in Fig. 3. In this figure, the thick solid lines show the cyclic stress-strain curves obtained by the incremental step tests ${ }^{(19)}$, and monotonic stressstrain curves are also shown for comparison. The initial part of the cyclic stress-strain curve of the [410] crystal is deliberately drawn by a thin line because the initial part of the curve largely deviates from the plot of the saturation stress. The part of the cyclic stress-strain curves drawn by bold lines fit with the plot of the saturation stress. The cyclic stress-strain curve of the [410] crystals is different from that of the [110] crystal, while the monotonic stressstrain curves of the [410] and [110] crystals were similar in the eary stage of deformation as seen in Fig. 3.

Figure 4 shows a $\log$-log plot of the plastic strain amplitude versus the number of cycles to failure. The relation between the plastic strain amplitude and cycles to failure approximately satisfied the Manson-Coffin's relationship ${ }^{(20)}$.

\section{Shape change during cyclic deformation}

During cyclic deformation, a part of the gage section of the [110] crystals thinned down and the reminder in the gage section thickened as shown in Photo. 1. This tendency started 


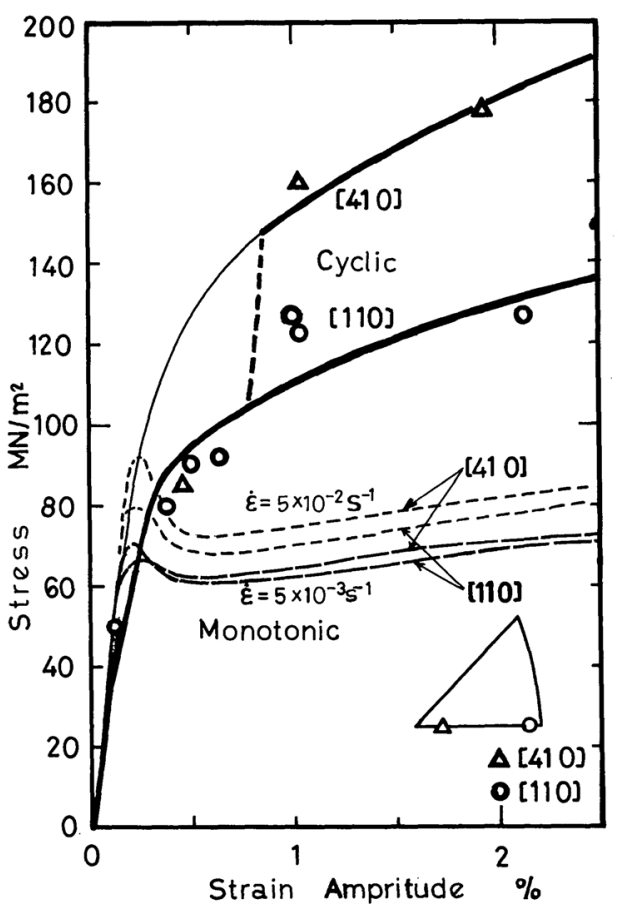

Fig. 3 The saturation stresses are plotted against total strain amplitude for the [110] and [410] crystals. Cyclic stress-strain curves obtained by a covenient method $^{(19)}$ also shown by bold lines in the range where the curve fit with the plot of the saturation stress. The monotonic stress-strain curves of both crystals are shown for comparison.

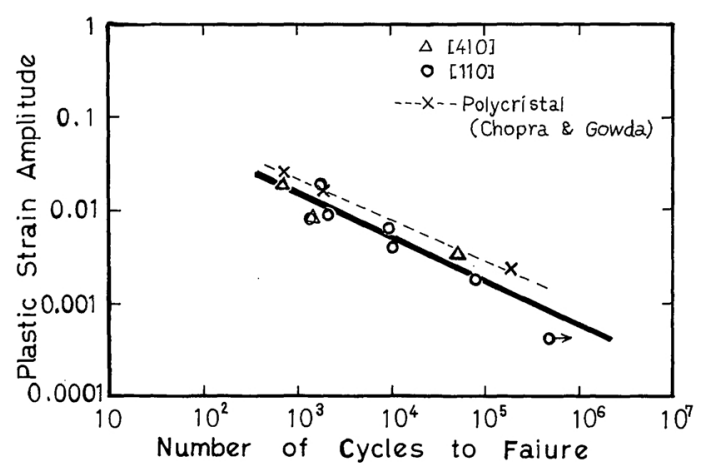

Fig. 4 The log-log plot of the plastic strain amplitude vs number of cycles to failure.

before the stress amplitude attained its saturation stress. Cracks initiated at pronounced slip bands which appeared in the thinned region of the specimen. In the [410] crystals the shape change was not very severe compared with the [110] crystals. In most of the specimen of the

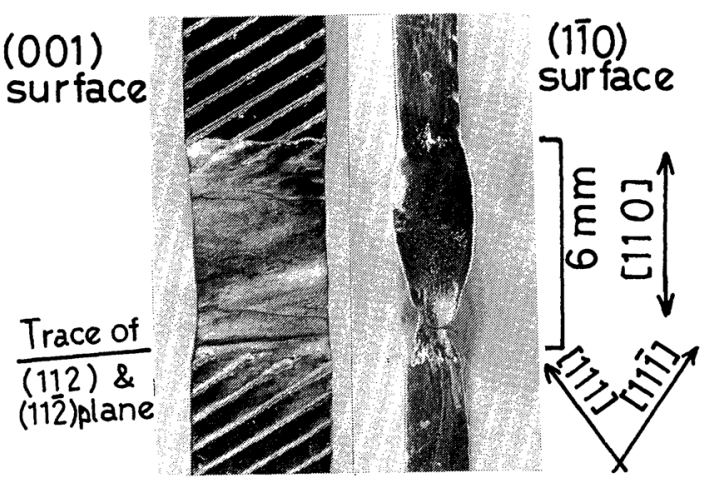

Photo. 1 The final shape of a fatigued [110] crystal.

[410] crystal, cracks started in the region near the grips.

\section{Dislocation distribution}

Thin foils were cut from the places apart from the fracture surface in the fatigued and fractured specimens and dislocation distributions were observed. Some foils were made from the unfractured specimens. Photograph 2 shows the dislocation distribution in the [110] specimens cycled at small plastic strain amplitudes; (a) shows a foil of the (001) section when the plastic strain amplitude was $0.04 \%$, and (b) the (110) section when the plastic strain amplitude was $0.17 \%$. Dense clusters of small loops or edge dipoles were seen in the crystals. These clusters were frequently elongated along the slip plane. Two kinds of screw dislocations with different Burgers vectors are seen in the micrograph. Small dislocation loops or black spots were seen between the clusters. Photograph 3 shows cell structures formed in the [110] crystal cycled at high strain amplitudes: (a) shows a foil of the (001) section when the plastic strain amplitude was $0.39 \%$, and (b) shows a foil of the (110) section when the plastic strain amplitude was $0.52 \%$. These cell walls were not parallel to the slip plane. It should be remembered that cell walls formed in a crystal deformed in a monotonic manner are approximately parallel to the $\{112\}$ slip plane ${ }^{(21)}$.

Photograph 4 shows a typical dislocation distribution in the [410] crystal cycled at a small plastic strain amplitude by $0.20 \%$. The micrograph was taken of the foils parallel to the $(01 \overline{1})$ plane, which was inclined from the 

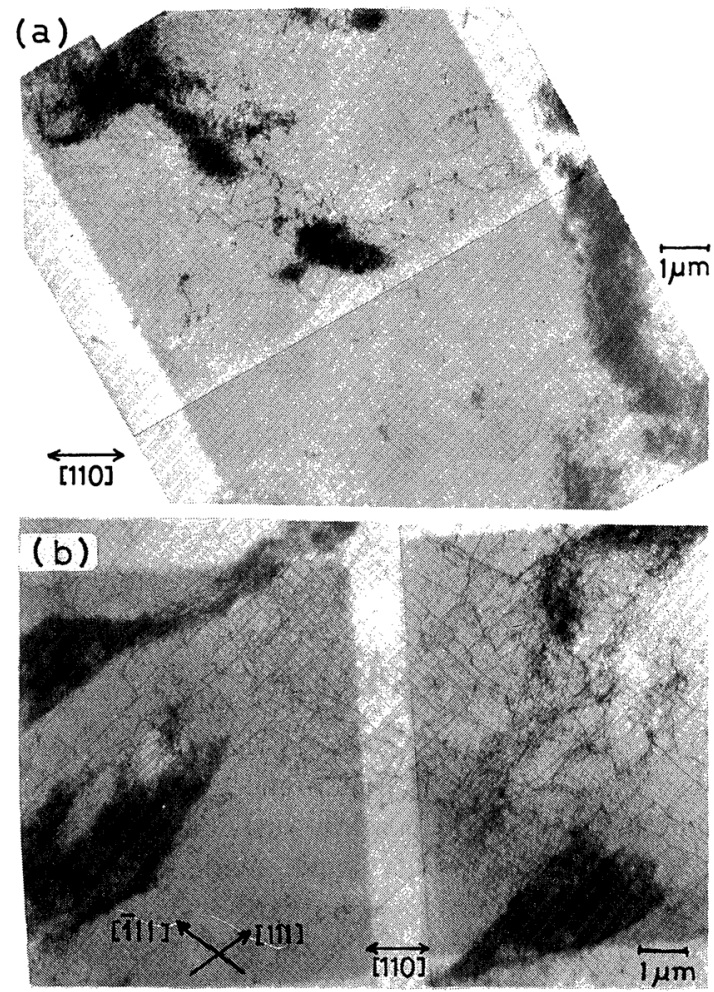

Photo. 2 Dislocation distributions in the [110] crystal after being cycled at small strain amplitudes. (a) is the (001) foil, after $4.7 \times 10^{5}$ cycles at the plastic strain amplitude of $0.04 \%$. (b) is the (110) foil after $9 \times 10^{4}$ cycles at a plastic strain amplitude of $0.17 \%$.

stress axis by 13 degrees. The majority of the cluster was parallel to the [111] slip direction but some were parallel to the [11̄i] direction as shown by $\mathrm{A}$ in Photo. 4. Some clusters, for example B, were deemed parallel to the [100] axis, which was approximately parallel to the stress axis. The existence of the isolated clusters like $A$ and $B$ is emphatically shown in the micrograph. An observation over a wide area showed that more than $70 \%$ of the area was covered by the dispersed clusters parallel to the [111] direction. A cluster free zone usually existed around the isolated cluster.

Photograph 5 shows the dislocation distributions in the [410] crystal fatigued at a large plastic strain amplitude by $0.82 \%$. In this micrograph, cell walls look not parallel to the slip plane but roughly parallel or normal to the [100] direction. They must have a tendency

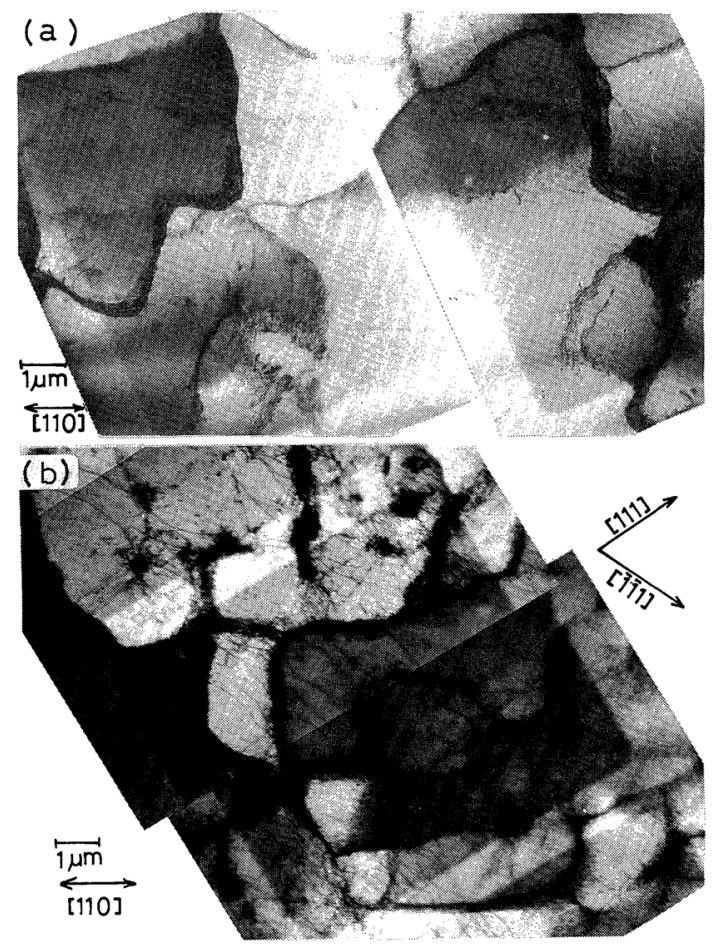

Photo. 3 Dislocation distributions in the [110] crystal after cyclic deformation at large strain amplitudes. (a) is the (001) foil after $1.1 \times 10^{4}$ cycles at the plastic strain amplitude of $0.39 \%$. (b) is the (110) foil after $9.8 \times 10^{3}$ cycles at a plastic strain amplitude of $0.52 \%$.

to lie on a $\{100\}$ plane because tangles in the [100] crystal fatigued at $-78^{\circ} \mathrm{C}$ have a strong tendency to lie on a $\{100\}$ plane $^{(22)}$. At least three and probably four kinds of screw dislocations with different Burgers vectors exist in the crystal, as seen from the fact that screw dislocations $\mathrm{A}, \mathrm{C}$ and $\mathrm{D}$ lie along the different directions in Photo. 5.

\section{Fracture surface}

A typical fracture surface of the [110] crystal is shown in Photo. 6, where (a) is a high magnification micrograph in the area denoted by an arrow in (b). Fatigue cracks were always nucleated on the (001) surface for the [110] specimen, where slip steps are made only on the (001) surface as seen in the geometrical relation shown in the inset in Photo. 6. Striation patterns were generally parallel to the intersection between the operative $\{112\}$ slip planes 


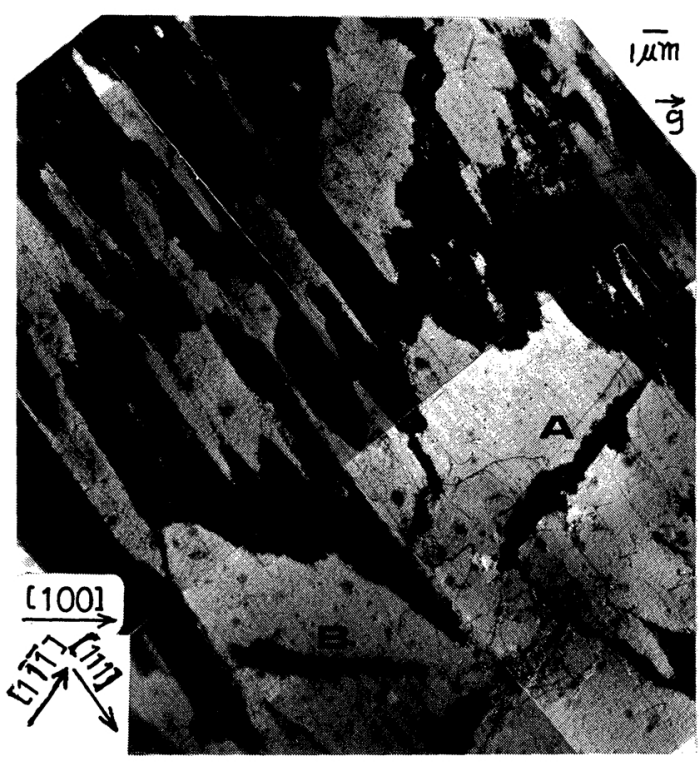

Photo. 4 A dislocation distribution in the [410] crystal after cycled at a small plastic strain amplitude of $0.20 \%$ by $4.6 \times 10^{5}$ cycles. The foil surface is $(01 \overline{1})$. The projection of the [410] direction on this micrograph is close to the [100].

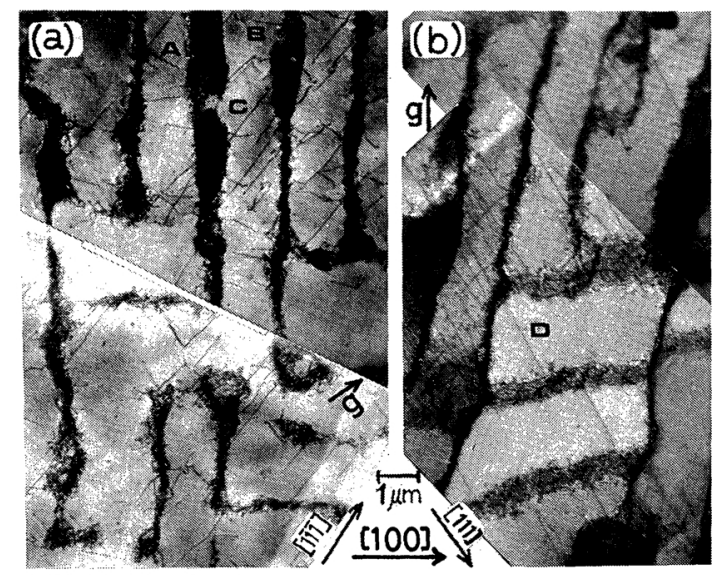

Photo. 5 Dislocation distributions in the [410] crystal after cycled at a large plastic strain amplitude of $0.82 \%$ by $1.4 \times 10^{3}$ cycles. Foil surface is $(01 \overline{1})$. (a) and (b) show different places in the same specimen and were taken by different $g$ vector. Projection of the [410] direction on this micrograph is close to the [100].

and the (110) fracture surface, when the macroscopic fatigue fracture surface is normal to the stress axis.

Fracture surface of the [410] crystal was
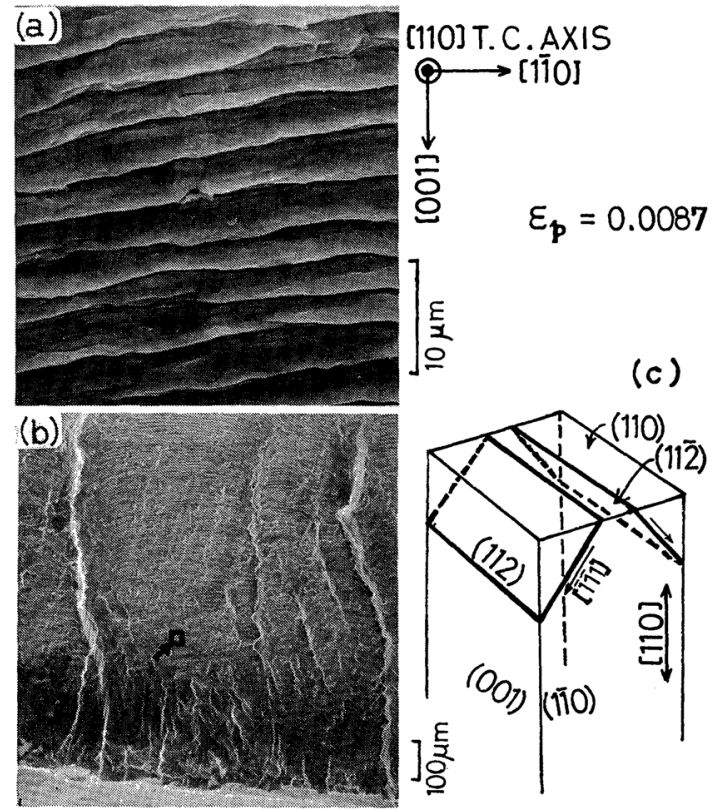

Photo. 6 A typical fracture surface of the [110] crystal after cycled at a strain amplitude of $0.87 \%$. (a) is a high magnification micrograph in the area denoted by an arrow in (b). (c) shows a geometrical relation between the specimen surface and operative slip systems.

rugged as shown in Photo. 7. Main surface steps were roughly parallel to the slip traces of $\{112\}$ slip planes.

An additional test was made on the [110] crystal to clarify whether or not a crack can propagate along the direction perpendicular to the operative slip vectors. A notch $(1.5 \mathrm{~mm}$ deep, $0.5 \mathrm{~mm}$ wide) was introduced perpendicular to the (110) surface in a pre-deformed [110] crystal $(3 \times 8 \times 24 \mathrm{~mm})$ as schematically shown in Fig. 5. The specimen was predeformed by $25 \%$ of unidirectional strain at $-78^{\circ} \mathrm{C}$ so that the fatigue crack nucreate easier than in annealed crystal at the notch tip. Under the uniaxial stress along the [110] direction, operative slip vectors do not have a component in the [110] direction along which a crack is expected to propagate. The specimen was cycled in push-pull under the grip distance control by an amplitude of $60 \mu \mathrm{m}$ in $2 \mathrm{~Hz}$, where the initial grip distance was $6 \mathrm{~mm}$. The nominal stress amplitude was about $83 \mathrm{MN} / \mathrm{m}^{2}$ in the initial stage of crack propagation. A crack 

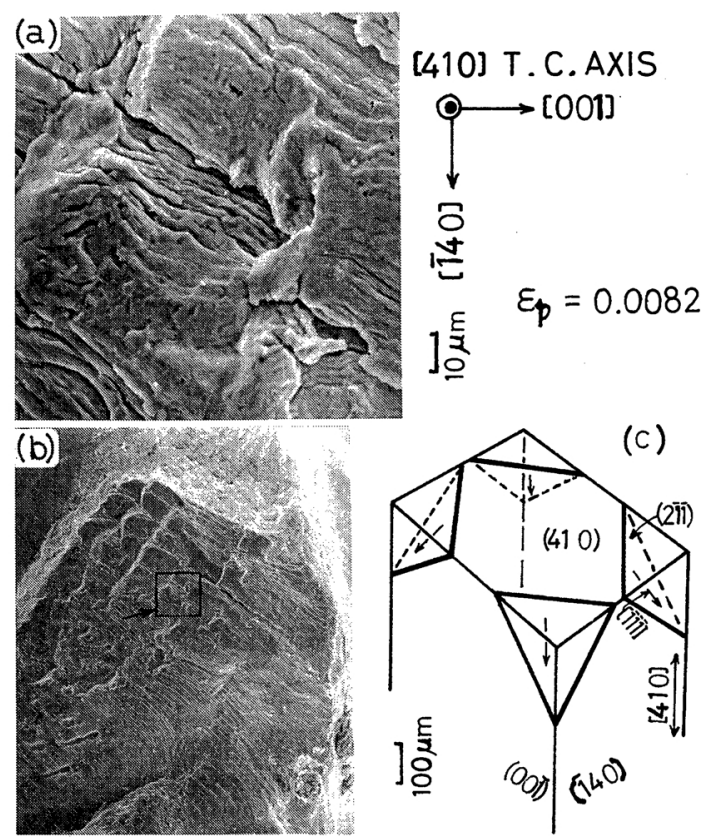

Photo. 7 A typical fracture surface of the [410] crystal after cycling at a strain amplitude of $0.82 \%$. (a) is a high magnification micrograph in the area denoted by an arrow in (b). (c) is a geometrical relation between the specimen surface and operative slip systems.

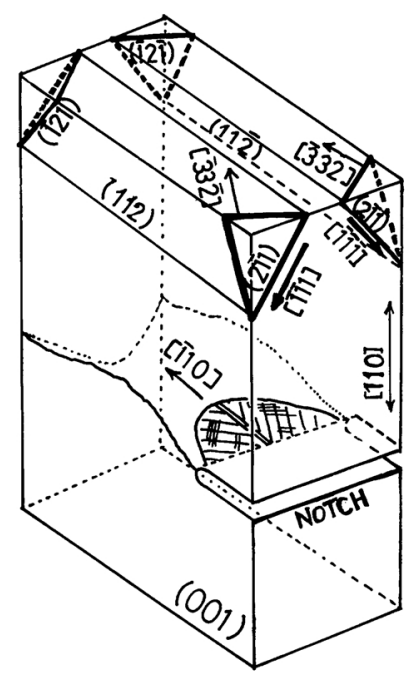

Fig. 5 The specimen to check whether the fatigue crack propagates to normal to the operative slip vectors. Operative $\{112\}$ slip planes, operative $\langle 111\rangle$ slip vectors, a notch and schematic of the fracture surface are shown. initiated at the center of the notch tip and propagated on the (110) plane as shown in Photo. 8. The hatched region schematically shown in Fig. 5 corresponds to the region of leaf-like pattern grown from the notch root shown in Photo. 8(a). The macroscopic fracture surface of this region was perpendicular to the stress axis. This region almost satisfied the plain strain condition for crack propagation ${ }^{(1)}$. The fracture surface outside this region was not perpendicular to the stress axis, corresponding
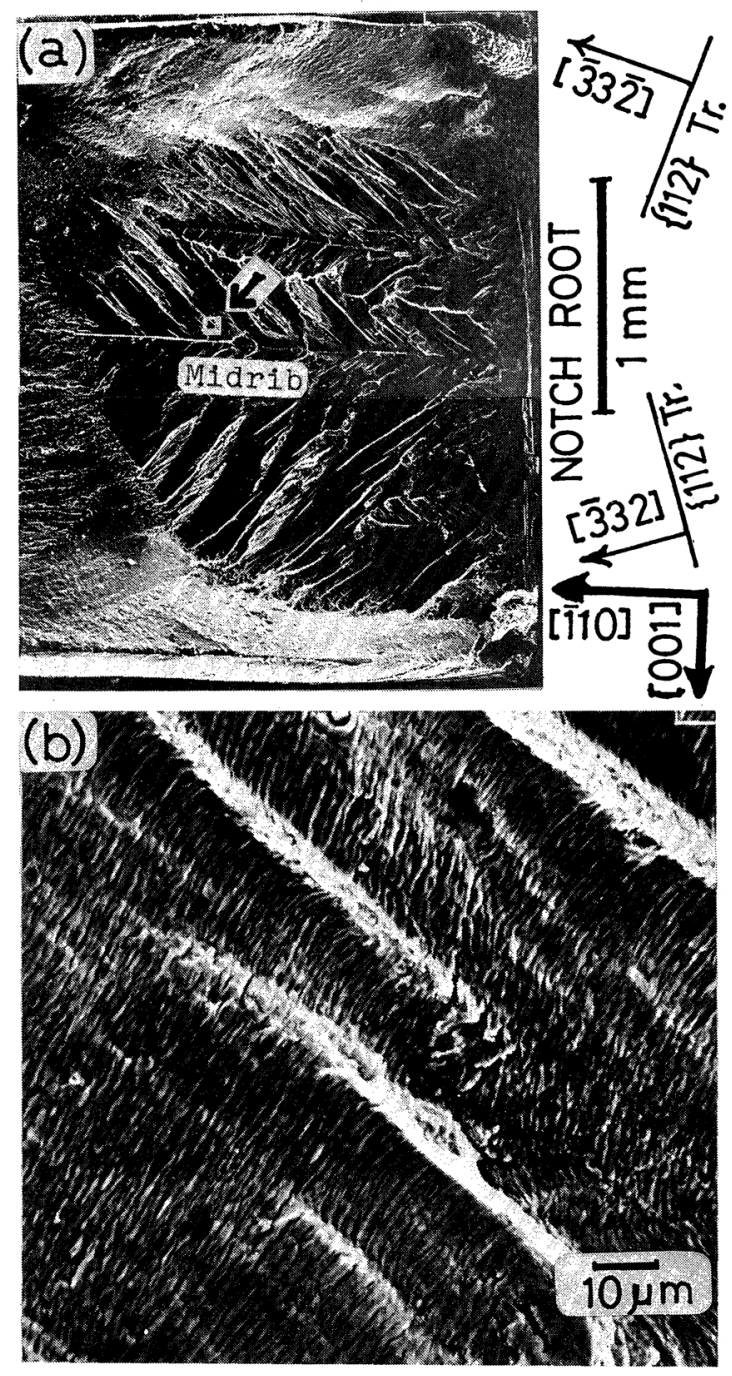

Photo. 8 A fracture surface of the [110] specimen when a crack was started at the notch root introduced from the (110) plane, and propagated to the interiour. (b) is a high magnification micrograph in the area denoted by an arrow in (a). 
to the plane stress condition for crack growth. The leaf-like region can be divided into two regions. Each region had a striation mark with different directions. Photograph 8(b) shows an enlarged micrograph of a framed part in (a). As seen in this micrograph, the striation marks, which indicate the normal direction of fatigue crack growth $^{(1)}$, are not parallel to the notch tip but parallel to the traces of the (211) and (121) slip planes. The striation pattern in the region below the midrib in Photo. 8(a) was parallel to the trace of the (211) and (121) slip planes. The midrib is composed of a sharp step in which the side surface between the plateaus is approximately parallel to the (001) plane.

\section{Discussion}

\section{Dislocation substructure corresponding to strain amplitude and crystal orientation}

The dislocation substructure in iron generated by cyclic deformation has been observed by several authors using polycrystals ${ }^{(23)(24)}$ and single crystals oriented for single slip ${ }^{(12)(16)}$. The substructure in fatigued iron generally depends on the applied strain range. In a low strain range, dislocations were primarily in the form of edge dipoles, clusters of loops and tangles besides isolated screw dislocations, whereas a cell structure is developed in a high strain range.

Krejci and Lukas ${ }^{(12)}$ fatigued iron single crystals oriented for single slip with a frequency of $105 \mathrm{~Hz}$ and a stress amplitude of $118 \mathrm{MN} / \mathrm{m}^{2}$ over $80 \%$ of the expected lifetime, and showed that the tangles are formed along one of the $\langle 111\rangle$ Burgers vectors. In the present investigation, when the strain amplitude is small, the most of clusters locally parallel to one of the $\langle 111\rangle$ slip direction even through the specimen axis was oriented for the multiple slip deformation as seen in Photos. 2 and 4. This fact suggests that the single slip is locally predominant in the [110] and [410] crystals when the strain amplitude is low. On the contrary, cell walls developed at a high strain range have a tendency parallel to a $\{110\}$ or $\{100\}$ plane as shown in
Photos. 3 and 5. These planes of the cell walls have an equivalent orientation relation with two and four active $\{112\}$ slip systems in the [110] and [410] crystals, respectively. This substructure must make even contribution to each slip system as the origin of hardening. This suggests that the two and four slip systems are comparatively equally activated in all over the crystal when the strain amplitude is large in the [110] and [410] crystal, respectively. It would be expected from the result of the monotonic deformation that the four fold slip brings a large work hardening but the single slip brings about low work hardening. The discontinuity of the cyclic stress-strain curve of the [410] crystal around the $0.5 \%$ strain amplitude must be due to the change of the number of the operative slip systems. In the [110] crystal, the plotted stress at about $1 \%$ strain is abnormally higher than that at about $0.5 \%$. This gap must results from the difference of the dislocation substructure as shown in Photos. 2 and 3.

\section{A comparison between cyclic and monotonic deformation}

The dislocation substructure generated by the cyclic deformation will be compared with that by the monotonic extension under the condition of the same level of the stress increment from the yield stress. When the strain amplitude is $0.2 \%$, the stress increment is about $20 \mathrm{MN} /$ $\mathrm{m}^{2}$, and the same increment can be obtained by $3 \%$ extension. The corresponding dislocation substructure in iron of the [110] and [100] crystal generated by monotonic deformation is composed of the diffuse clusters of dislocation loops and tangles of dislocations besides screw dislocations as seen in Figs. 7 and 9(a) in the previous paper ${ }^{(21)}$. On the other hand, the dislocation density in the cluster and tangles generated by cyclic deformation is much higher than that generated by monotonic extension as shown in Photos. 2 and 4 in the present paper. The comparison suggests that the volume fraction of the loop cluster does not show any remarkable effect on the stress increment. As a matter of fact, even when the volume fraction of the loop cluster exceeds $50 \%$ in total volume of the [410] crystal as shown in Photo. 4, the increment of the flow stress is only $20 \mathrm{MN} / \mathrm{m}^{2}$. 
It is considered that the cluster parallel to the slip plane is composed of edge dipoles ${ }^{(1)}$, so that the crystal occupied by the cluster is deformed during cycles by flip-flop motion of edge dipoles ${ }^{(1)}$. If dislocations in the dense cluster are fixed and this region deforms only by elastic, $0.2 \%$ of the applied plastic strain makes $400 \mathrm{MN} / \mathrm{m}^{2}$ in the region (Young's modulus is assumed to be $200 \mathrm{GN} / \mathrm{m}^{2}$ ), which is considerably higher than the observed stress of $20 \mathrm{MN} / \mathrm{m}^{2}$.

The major slip direction is believed to be parallel to the [111] direction in the crystal, as shown in Photo. 4, by analogy with the crystal oriented for single slip ${ }^{(12)}$. Tangles like $\mathrm{A}$ and B have different geometrical direction with the major slip direction, so that they must not be a simple cluster of edge dislocation dipoles but tangles with dislocations of different Burgers vectors. The wide cluster free zone around these tangles seems to indicate the existence of the long-range stress field under stress.

The dislocation substructure of the [100] crystal deformed by $19.6 \%$ in a monotonic manner is shown in Fig. 10 in the previous paper $^{(21)}$. The stress increment of this crystal, about $90 \mathrm{MN} / \mathrm{m}^{2}$, is about the same as that of the cycled crystal shown in Photo. 5. The density of the cell walls in the both crystals is also about the same, though the density of screw dislocations between the cell walls of the extended crystal is three times as large as that in the cycled crystal. Therefore, it is very likely that the strength of these crystals mainly depends on the density of cell walls compared with that of dislocations distributed between the cell walls.

\section{Shape change during cyclic deformation on the $[110]$ crystal}

A mechanism for the shape change under cyclic deformation has been reported by Nine (13), Neumann ${ }^{(15)}$, and Mughrabi and Wuthrich ${ }^{(17)}$, showing that the main origin is the anisotropy of the flow stress for screw dislocations in bcc metals. This mechanism for the shape change is available when the specimen is oriented for single slip. However, the mechanism cannot be applied in the present investigation of the [110] specimen because the specimen has a double slip orientation. Alternative mechanism is necessary to explain the necking and thickening during cycling.

\section{Fracture surface}

Neumann ${ }^{(4)}$ observed fracture surface of fatigued single crystals of copper and showed that when the stress axis is $\{100\}$ the fatigue crack is macroscopically flat but is rugged when the stress axis is not $\{100\}$. Concerning to $b c c$ metals, fatigue test of $\mathrm{Ti}-\mathrm{V}$ alloy were recently made by Carlson and $\mathrm{Koss}^{(18)}$. They showed that the fracture surface of the [100] specimen was rugged but that of the [110] was macroscopically flat. Their result is consistent with that of the present investigation (Photos. 6 and 7). In iron crystals, most of the striation marks on the fracture surface are parallel to the intersection of a pair of the active $\{112\}$ slip plane, as shown in Photos. 6 and 7. The easiest propagation of fatigue crack is the [001] direction in the [110] crystal. In this case, the most favorable slip systems are [111] (112) and

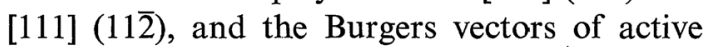
slip have the largest component in the propagation direction on the crack plane. The geometrical configuration is an ideal case for the Neumann's model ${ }^{(25)}$ of fatigue crack propagation.

The opposite case is that a crack is forced to propagate on the (110) plane in the [110] direction (see Fig. 5). In this case, no slip planes fit with Neumann's model ${ }^{(26)}$, so that the local propagation directions is $[\overline{3} 3 \overline{2}]$ or [ 332$]$ as shown in Photo. 8. The [111] ( $\overline{1} 2 \overline{1})$ and

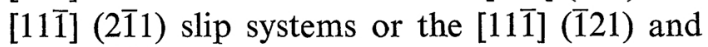
[111] (211) slip systems operate for the propagation in spite of their small Schmid factor.

A special feature in the crack growth along the [110] direction is the midrib in the leaf-like pattern. The striation patterns are symmetrical with respect to the midrib, which is precisely on the trace of the (100) plane, the cleavage plane in brittle fracture. At the crack front near the midrib, the mass transport under the tension cycle is in the $\pm[001]$ direction, (note that active Burgers vectors lie in the (110) plane), and a large tensile internal stress in the [001] axis appeares at the tip of the midrib; this stress may propagate the (001) crack in the 
[110] direction. The growth of the cleavage type (001) crack composes the leading front of the fatigue crack growing approximately in the [110] direction.

\section{Summary}

Single crystal of iron with the [110] and [410] axes were fatigued in push-pull at constant strain amplitudes in the range between 0.1 and $2 \%$ at room temperature.

(1) At a low strain amplitude $(<0.5 \%)$ the cyclic hardening of the [410] crystals was similar to that of the [110] crystal. Clusters of edge dipoles and loops are developed mainly parallel to a $\{112\}$ slip plane in both crystals. In the [410] crystal one slip system was active locally.

(2) At a high strain amplitude $(>0.5 \%$ ) cyclic hardening of the [410] crystals was larger than that of the [110] crystal. Cell structure developed extensively in both crystals. In the [410] crystal, four slip systems were active.

(3) The fatigue crack propagates most smoothly on the (110) plane in the [001] direction. When the crack is forced to propagate in the [110] direction, the local propagation direction is $[\overline{3} 3 \overline{2}]$ or $[\overline{3} 32]$.

\section{Acknowledgments}

The author would like to thank $T$. Takeuchi for his helpful comments on the manuscript. He also supplied a sheet of single crystal used in the present investigation.

\section{REFERENCES}

(1) J. C. Grosskreutz: Phys. Status Solidi, (b) 47
(1971), 11, 357.

(2) P. J. Woods: Phil. Mag., 28 (1973), 155.

(3) A. T. Winter: Phil. Mag., 30 (1974), 719.

(4) P. Neumann: Acta Met., 22 (1974), 1155.

(5) J. M. Finney and C. Laird: Phill. Mag., 31 (1975), 339.

(6) A. T. Winter: Phil. Mag., 31 (1975), 411.

(7) J. G. Antonopoulus and A. T. Winter: Phil. Mag., 33 (1976), 87.

(8) J. G. Antonopoulus, L. M. Brown and A. T. Winter: Phil. Mag., 34 (1976), 549.

(9) A. T. Winter: Phil. Mag., 37 (1978), 457.

(10) A. Yoshikawa and M. Okamoto: Proc. Int. Conf. on Strength of Metals and Alloys, Tokyo, Supplement to Trans. JIM, Vol. 9 (1968), p. 471.

(11) F. V. Lawrence, Jr. and R. C. Jones: Met. Trans., 1 (1970), 367.

(12) J. Krejci and P. Lukas: Phys. Status Solidi, (a) 5 (1971) 315.

(13) H. D. Nine: Phil. Mag., 26 (1972), 1409.

(14) M. Doner, J. C. Diprimo and E. I. Salkovitz: Acta Met., 21 (1973), 1547.

(15) P. Neumann: Z. Metallk., 66 (1975), 26.

(16) H. Mughrabi, K. Herz and X. Stark: Acta Met., 24 (1976), 659.

(17) H. Mughrabi and Ch. Wuthrich: Phil. Mag., 33 (1976), 963.

(18) J. A. Carlson and D. A. Koss: Acta Met., 26 (1978), 123.

(19) R. W. Landgraf, J. Morrow and T. Endo: J. Materials, 4 (1969), 176.

(20) L. F. Coffin, Jr.: Internal Stress and Fracture in Metals, Elsevier Pub. N.Y. (1959), p. 363.

(21) S. Ikeda: J. Phys. Soc. Japan, 27 (1969), 1564.

(22) S. Ikeda: Proc. 5th Intern. Conf. Strength of Metals and Alloys, Aachen, (1979) in press.

(23) J. T. Mcgrath and W. J. Bratina: Phil. Mag., 11 (1965), 429.

(24) O. K. Chopra and C. V. B. Gowda: Phil. Mag., 30 (1974), 583.

(25) T. Takeuchi: J. Phys. Soc. Japan, 27 (1969), 436.

(26) P. Neumann: Z. Metallk., 58 (1967), 780. 